the previous session. Therapists offer support to each other and collaborate in management strategies.

\section{Personal perspective}

Before working at Peel, my experience of managing patients with borderline personality disorder had been generally negative: I often felt ill-equipped to deal with their behaviour and I was conscious that my management was not always consistent. My perceived lack of skill fuelled countertransference towards them.

I do not believe that DBT represents the gold standard in treatment for borderline personality disorder, but it has given me a framework from which to work and the results I have seen are positive. I was surprised at how quickly I was able to learn the theory of DBT and, like some other clinicians at Peel, I have received only brief training in the basic theory of DBT. However, I believe that with the support of more experienced peers, clinicians are able to draw on their previous mental health experience to offer the validation required in individual therapy and reinforce skills learnt in the group; this makes DBT a practical option for CMHTs, which can, with a little extra training, utilise a skill base already in place.

I had previously worked in CMHTs with no specific treatment programme, where lack of structure and guidance on how to manage borderline personality disorder frequently resulted in splitting within the team. I recall lengthy hospital admissions being used for want of any alternative. I have also worked in CMHTs which have had access to specialist programmes, one run on a psychodynamic basis and another based on a DBT model. I was not directly involved in either programme and cannot comment on the efficacy of the therapy itself, but I do recall a sense of detachment of these services from the CMHT. There was a tendency for clinicians immediately to refer patients with borderline personality disorder to these services, without thinking of a management plan themselves; if the patient were then to present in crisis to the CMHT, management could turn to panic and often resort to hospital admission. The holistic and multidisciplinary approach of a DBT programme based within, and run by, a
CMHT ensures all clinicians are exposed to the often demanding cases of borderline personality disorder and yet no group is overwhelmed by them. It also ensures that no clinician becomes deskilled in the management of such patients and provides the patients with vital continuity of care.

I have also been able to apply these new skills to other patients, such as those with eating disorders and substance misuse problems.

The peer group supervision was invaluable in gaining support and provided an opportunity to interact with other disciplines, one which I have often found lacking in other CMHTs.

The clinicians at Peel were keen to innovate and develop services; their sense of pride in this programme was tangible and well deserved. Perhaps it is this team approach which, at least in part, transfers to and benefits the patient group. Bearing in mind those treatments that have been shown to be effective, the determination of which of these to employ may be less important than the team developing a common approach with a sense of purpose, which in itself leads to successful treatment. I believe that DBT allows for such an approach and my time at Peel inspired me with hope that even a small service with relatively few resources and little extra funding can provide an effective treatment option for borderline personality disorder. CMHTs in the UK looking to develop their service would do well to consider a similar approach.

\section{References}

Linnehan, M. M. (1993a) Cognitive Behavioural Treatment of Borderline Personality Disorder. Guildford.

Linnehan, M. M. (1993b) Skills Training Manual for Treating Borderline Personality Disorder. Guildford.

Moran, P. (2002) The epidemiology of personality disorder. Psychiatry, 1, 8-11.

NIMHE (2003) Personality Disorder: No Longer a Diagnosis of Exclusion. Policy Implementation Guidance for the Development of Services for People with Personality Disorders. NIMHE.

Palmer, R. L. (2002) Dialectical behaviour therapy for borderline personality disorder. Advances in Psychiatric Treatment, 8, 10-16.

Peel Development Commission (2005) Peel Development Commission Annual Report 2004-05. Government of Western Australia.

\title{
The yield from national surveys of mental health
}

\section{Scott Henderson FRCPsych ${ }^{1}$ and Gavin Andrews FRCPsych ${ }^{2}$}

'Emeritus Professor, John Curtin School of Medical Research, Australian National University, Canberra, ACT 0200, Australia, email ashenderson@netspace.net.au

${ }^{2}$ Scientia Professor, School of Psychiatry, UNSW at St Vincent's Hospital, Sydney, NSW, 2010, Australia

\begin{abstract}
t least 21 countries have now carried out national surveys of mental health under the aegis of the World Health Organization's World Mental Health Surveys. This has meant interviewing some 157000 people in their homes. The countries are as varied as Australia, China,
\end{abstract}

Iran, six continental European nations, Nigeria, the UK and the USA (Andrews et al, 2001; Demyttenaere et al, 2004; Mohammadi et al, 2005). It is therefore timely to consider what this very large body of information has yielded and to what use it can be put, especially in relation to the costs 
and human resources expended in a field where unmet need is so conspicuous.

\section{Methods}

The surveys have many attributes in common. They were all undertaken to inform health policy. Most used the same standardised interview with the same (DSM-IV) diagnostic criteria (American Psychiatric Association, 1994) for the common mental disorders. The latter are anxiety, depressive disorders and alcohol or substance misuse. All surveys were conducted by lay interviewers and the data are derived solely from self-report. The morbidity described is categorical (present or absent) rather than dimensional, and refers to symptoms both in the previous 12 months and across the respondent's lifetime. Independent variables have usually been confined to age, gender, marital status and indicators of socio-economic status. Most surveys covered the age range 18-65 years, and only a minority included children or the elderly. Most included measures of disability and recent health service use.

\section{Findings}

There have been some consistent findings. The total prevalence rates for adults have been much higher than might be expected, with the median 12-month prevalence for all disorders being $12.2 \%$. The range, however, is considerable, from $4.3 \%$ in Shanghai to $26.4 \%$ in the USA. Anxiety and depressive disorders are more common in women and substance use disorders are more common in men. The median age at first onset of anxiety disorders is 11 years, of substance use disorders 20 years, and of mood disorders 30 years. Mental disorders are therefore disorders that begin in the young. The burden of disability, in terms of the number of days lost from work or family life, is very much greater than might have been expected and yet the majority of sufferers received no treatment. This is so in higher-income as well as lower-income countries, even though inexpensive and effective treatments exist.

Response rates ranged greatly, from $51 \%$ in Belgium to $88 \%$ in Colombia (Demyttenaere et al, 2004). Some of the differences in total prevalence may also depend on the number of disorders included in the interview. But there are some striking differences in the prevalence estimates of individual disorders. For example, the 12-month prevalence of depressive disorder is $9.6 \%$ in the USA, 6.6\% in Lebanon (conducted in 2002-03), 3.1\% in Japan, $1.7 \%$ in Shanghai and $0.8 \%$ in Nigeria. The survey in Iran (Mohammadi et al, 2005) reported lifetime rather than 12-month prevalence, which was only $4.3 \%$ for depressive disorder. When the US 1991-92 national survey was repeated in 2001-02, the prevalence of depressive disorder had increased from $3.3 \%$ to $7.1 \%$ and treatment rates from $12 \%$ to $20 \%$ (Compton et al, 2006).

\section{The value of the surveys}

What does this massive body of data mean? It shows that psychiatric morbidity is common, although it would be wrong to conclude that a 1 -year prevalence rate of $12 \%$ means that one in eight citizens of a country wish for or need treatment. Where the investigators have included a measure of disability, this has allowed a better estimate of unmet need. It has shown that the economic and social burden arising from mental disorders is highly significant for health policy. These two findings, the high prevalence and the proportion of all disability that comes from mental illness, have been the most influential products of the surveys. They have been noted by senior administrators and politicians, often accompanied by an increased allocation of funds for services and research. The increased prevalence of major depression observed in the USA shows the value of repeating surveys to monitor the mental health of a nation.

By contrast, the scientific advance in understanding the causes of these mental disorders has been slight. The gender differences in anxiety and depressive symptoms were already known. The data on age of first onset in community samples are new, but, that apart, no new hypotheses on aetiology of any fundamental significance have been generated. The psychoses, which are so disabling, are too low in prevalence for useful data on their aetiology to be obtained from a crosssectional survey in the community. For aetiological research, data on environmental risk factors and temperament could have been included, but it has proved difficult to persuade funding bodies to include these measures to inform aetiology when what they want are answers to three questions:

o How many people have which disorder?

How disabled are they?

What services do they need and want?

As a result, new information on causation has been sparse, despite the massive sample size.

It is always tempting to compare prevalence rates between countries. Where differences are observed, as in the above examples, it is tempting to put them down to intrinsic attributes of the population, such as lifestyle or social cohesion. However, the estimates must inevitably be affected by differences in method, such as the response rates in each survey, the use of different instruments or versions thereof, and the readiness of respondents in different countries to acknowledge the presence of psychological or physical symptoms. Also, it may be a mistake to assume the diagnostic criteria are equally applicable across countries. Four decades ago, in his influential paper 'Are international comparisons timely?', Kessel (1965) concluded that they were not and that epidemiological work on the aetiology of mental disorders would be better directed to within-country studies. We believe the situation remains the same today. It has to be concluded that the massive effort to obtain data on the mental health of nations, involving over 150000 respondents, has contributed little to the understanding of the causes of mental illness.

\section{Future surveys}

It is likely that some countries will repeat their national surveys and others will conduct their first. The justification must be largely to keep mental health on the political agenda and to monitor changes in service use. The use of standardised interviews, matched to ICD-10 (World Health Organization, 1992) and DSM-IV classifications (American Psychiatric Association, 1994) or their successors, will 
continue, because rates for such categorical diagnoses seem to have an administrative impact.

The scientific value of this categorical approach is less certain. Much greater scientific value will come from the analysis of dimensional scales such as the Kessler Psychological Distress Scale (K-10; Kessler et al, 2002) and 12-item General Health Questionnaire (GHQ-12; Goldberg \& Williams, 1988). Unlike the diagnostic interview, which can take over an hour, these take only a few minutes to administer, even for persons with limited literacy. The scales cover the full range of symptoms of epidemiological interest and they detect the sub-clinical level of morbidity that is of such relevance to disease burden. Their continuous scores avoid the loss of statistical information that comes with categorical diagnoses. They are also less open to the biases that make international comparisons of prevalence rates so difficult to interpret. As Rose (1993) emphasised, the distribution of scores can be used to characterise whole populations. The K-10 has been included in many of the surveys, but has not yet been used to test aetiological hypotheses.

Further large-scale epidemiology needs to contribute to knowledge of aetiology. For example, in addition to obtaining psychiatric measures and exposures to adversity, obtaining genetic data is now feasible. This would allow the study of interactions between genotype and environment in very large samples. National surveys of mental health are major epidemiological undertakings. Their yield can now be expanded.

\section{References}

American Psychiatric Association (1994) Diagnostic and Statistical Manual of Mental Disorders (4th edn) (DSM-IV). American Psychiatric Press.

Andrews, G., Henderson, S. \& Hall, W. (2001) Prevalence, comorbidity disability and service utilisation. Overview of the Australian National Mental Health Survey. British Journal of Psychiatry, 17, 145-153.

Compton, W. M., Conway, K. P., Stinson, F. S., et al (2006) Changes in the prevalence of major depression and comorbid substance use disorders in the United States between 1991-1992 and 2001-2002. American Journal of Psychiatry, 163, 2141-2147.

Demyttenaere, K., Bruffaerts, R., Posada-Villa, J., et al (World Mental Health Survey Consortium) (2004) Prevalence, severity, and unmet need for treatment of mental disorders in the World Health Organization World Mental Health Surveys. JAMA, 291, 2581-2590.

Goldberg, D. P. \& Williams, P. A. (1988) User's Guide to the GHQ. NFER Nelson.

Kessel, N. (1965) Are international comparisons timely? Milbank Memorial Fund Quarterly, 2, 199-212.

Kessler, R. C., Andrews, G., Colpe, L. J., et al (2002) Short screening scales to monitor population prevalences and trends in non-specific psychological distress. Psychological Medicine, 32, 959-976.

Mohammadi, M-R., Davidian, A., Noobala, A. A., et al (2005) An epidemiological survey of psychiatric disorders in Iran. Clinical Practice and Epidemiology in Mental Health, 1, 16-23.

Rose, G. (1993) Mental disorders and the strategies of prevention. Psychological Medicine, 23, 553-555.

World Health Organization (1992) The ICD-10 Classification of Mental and Behavioural Disorders. Diagnostic Criteria for Research. WHO.

\section{A re-evaluation of the yield from national surveys of mental health}

\section{Ronald C. Kessler $\mathrm{PhD}^{1}$ and T. Bedirhan Üstün $\mathrm{MD}^{2}$}

'Department of Health Care Policy, Harvard Medical School, email kessler@hcp.med.harvard.edu ${ }^{2}$ Classifications, World Health Organization

$\mathrm{H}$ aving spent a considerable amount of time thinking about the uses of large-scale descriptive psychiatric epidemiological needs assessment surveys in our capacity as co-directors of the World Health Organization's World Mental Health Survey Initiative, we agree with many of the conclusions of Henderson and Andrews. Most importantly, we agree:

that among the most important benefits of these surveys have been their political value in documenting high prevalence and high disability

o that the time has come to expand the focus to study causes.

Preparation of this commentary was carried out in conjunction with the WHO World Mental Health (WMH) Survey Initiative, which is supported by the US Public Health Service (R01-MH070884, R13-MH066849, R01-MH069864, and R01-DA016558), the Fogarty International Center (FIRCA R01-TW006481), the Pan American Health Organization, the John D. and Catherine T. MacArthur Foundation, the Pfizer Foundation, Eli Lilly and Company, Ortho-McNeil Pharmaceutical, GlaxoSmithKline, and Bristol-Myers Squibb. More information about WMH is available at http://www.hcp.med.harvard.edu/wmh. The views expressed in this article are those of the authors and do not necessarily represent the official position of the World Health Organization, Harvard Medical School, or any of the organisations that fund the WMH research.
However, we also disagree with Henderson and Andrews on several points.

The first paragraph of their article raises a concern that the resources used to carry out psychiatric epidemiological surveys might be better used to address the problem of unmet need for treatment. This criticism is ill-conceived. These surveys make it clear that an increase in healthcare resources is required to address the enormous problem of unmet need for treatment of mental disorders. Needs assessment surveys must be carried out to document this unmet need, as well as to track changes in unmet need as mental healthcare policies change. It is a mistake to set the cost of carrying out these surveys in opposition to the need for increased treatment resources. This is especially so in light of the fact that the resources needed to carry out a typical large-scale psychiatric epidemiological survey (e.g. face-to-face interviews with a representative sample of 5000 respondents) are trivial in relation the resources needed to provide treatment. The former resources amount to about 25 person-years. In a country of 40 million people with a $15 \%$ prevalence of mental illness, this comes to roughly 1 minute per person with a mental illness. Not much good 